\title{
Tunable Multilayers of Self-Organized Silica Nanospheres by Spin Coating
}

\author{
Sofiane Kasmi, ${ }^{1}$ Jeanne Solard, ${ }^{2,3}$ Inga Tijunelyte, ${ }^{1}$ Alexis P. A. Fischer, ${ }^{2,3}$ \\ Marc Lamy de la Chapelle, ${ }^{1}$ and Nathalie Lidgi-Guigui $i^{1}{ }^{1}$ \\ ${ }^{1}$ Laboratoire CSPBAT UMR CNRS 7244, UFR SMBH, Université Paris 13, Sorbonne Paris Cité, 74 rue Marcel Cachin, \\ 93017 Bobigny, France \\ ${ }^{2}$ Laboratoire de Physique de Lasers CNRS UMR 7538, Université Paris 13, Sorbonne Paris Cité, 99 avenue Jean-Baptiste Clément, \\ 93430 Villetaneuse, France \\ ${ }^{3}$ Centrale de Proximité en Nanotechnologies de Paris Nord, Université Paris 13, Sorbonne Paris Cité, 99 avenue Jean-Baptiste Clément, \\ 93430 Villetaneuse, France
}

Correspondence should be addressed to Nathalie Lidgi-Guigui; nathalie.lidgi-guigui@univ-paris13.fr

Received 24 November 2017; Revised 1 February 2018; Accepted 12 February 2018; Published 14 March 2018

Academic Editor: Weidong Liu

Copyright (C) 2018 Sofiane Kasmi et al. This is an open access article distributed under the Creative Commons Attribution License, which permits unrestricted use, distribution, and reproduction in any medium, provided the original work is properly cited.

\begin{abstract}
The coating of fused silica by an organized layer of silica nanospheres (NS) is an important issue for the design of optical and topographic properties especially for lithography techniques such as nanosphere lithography (NSL) or nanosphere photolithography (NSPL). Here, the spin coating of NS dispersed in N,N-dimethylformamide (DMF) is studied. The role of the NS diameter, the spin-coating acceleration, and the volume fraction are the parameters to take into account for the formation and organization of NS in single or double closely packed layers. We propose an explanation for this behavior based on the transition between sedimentation and a viscous regime on the basis of the silica NS organization.
\end{abstract}

\section{Introduction}

The organization of nanostructures on a surface is an important issue that many fields are confronted with. The application field of such materials depends on the dimension range of the nanostructures. For this purpose, a large variety of techniques have been developed to nicely localize the structure at a desired position. Top-down techniques, such as lithography, are preferred when it comes to placing a single structure at a specific position. For instance, e-beam lithography (EBL) uses the electron beam of a scanning electron microscope (SEM) to draw a pattern on an electrosensitive resist. If EBL can indeed be used to design assemblies of nanostructures, it will be highly time-consuming and will become quite challenging when more than $1 \mathrm{~mm}^{2}$ has to be covered. For large assemblies of nanostructures, soft lithography techniques can be exploited such as nanoimprint lithography $[1,2]$. Yet, bottom-up techniques are often more suitable for surface functionalization [3, 4], which make nanostructures become trapped on the surface by reactive termination. Another strategy more suitable for larger nanostructures (a few hundreds of nanometers) is to deposit a drop of the solution in which nanospheres (NS) are dispersed; it has been shown that the evaporation of the solvent allows the formation of a nicely organized film [5]. However, this technique is difficult to control especially for samples with an area typically greater than $1 \mathrm{~mm}^{2}$. This is why other techniques have been developed with success to reach the objective of the elaboration of a single layer of NS on a large area: Langmuir-Blodgett [6, 7], surface chemistry [8], and dip coating $[9,10]$. Spin coating is probably the easiest and fastest way to get to this result [11-14]. It consists in placing a droplet of an NS solution on a substrate and taking advantage of its fast rotation to spread the liquid. In order to form a layer of organized NS, they have to be dispersed in a solution with high wettability (so that they can easily spread on the surface) and high volatility (so that the liquid phase is quickly removed as soon as the NS are spread). The wettability is usually achieved by adding a surfactant such as octoxynol, while the volatility is achieved by choosing 
a solvent such as methanol. As the solution result in a mixture of surfactant, solvent, and NS, a fine optimization procedure has to be implemented. Octoxynol in methanol is one of the most frequent combinations reported in the literature; its concentration ratio can vary from a reference to another $[15,16]$. This demonstrates that the reproducibility of the results is rarely straightforward. Choi et al. [11] have proposed the use of N,N-dimethylformamide (DMF) as a solvent, as it has all the necessary qualities to easily and efficiently perform deposition of a large area of self-organized NS. Most importantly, as DMF is used in a pure form, no optimization step is required.

The use of single or double layers can have a tremendous advantage in a diversity of topics. For example, soft lithography techniques are currently developed to promote nanostructuration on transparent and flexible substrates. Nanoimprint lithography (NIL) $[1,2]$, nanosphere lithography (NSL), $[14,16]$ and its derivative Film Over Nanospheres (FON) [15] or nanosphere photolithography (NSPL) [10] can all take advantage of the ease of controlling the number of silica NS layers. In these techniques, the pattern formed by the organization of the NS is used as a mask or a mold. In NSL, metal is deposited through the spaces in between the NS; it forms triangles for a single layer of NS; more complex patterns can be reached with a two-layer mask. In NSPL, silica NS are used as lenses for UV light illumination of photoresists. This technique can also take advantage of varying the number of NS layers in order to create different patterns of interferences. For these particular applications, the number of layers should not be too high so a nanostructure can still be formed (presence of interstice for NSL and transmission for NSPL).

In this paper, we study the behavior of NS solution in DMF when spread onto silica surfaces. These surfaces are very similar to the oxide layer of silicon that can be found on top of silicon wafers. Furthermore, the silica surfaces that we have used in this work are transparent to UV and visible light. These substrates can thus be used in a wide range of applications such as transparent electronic devices or as photolithographic masks as proposed by Ayenew et al. [10]. The study of the organization of NS on silica surfaces shows that it is possible to control the number of NS organized layers using a spin-coating technique. We have considered the diameter of the NS and their concentration; these intrinsic properties of the solution are decisive to explain how the spreading occurs in a spin-coating experiment. In particular, the acceleration of spinning has an influence on the organization of the NS. By carefully choosing these parameters, one can choose to form either a single or a double layer of closely packed NS.

\section{Experimental}

DMF was purchased from Carlo Erba. Silica powders of NS with diameters of $310 \mathrm{~nm}$ and $540 \mathrm{~nm}$ were purchased from Polysciences Europe GmbH. Solutions of both powders were prepared with volume fractions of $4.1 \%, 6.1 \%, 7 \%$, and $7.9 \%$. The volume fraction $V_{f}$ is calculated as follows:

$$
V_{f}=\frac{4}{3} \pi R^{3} \frac{N}{V}
$$

where $R$ is the radius of the NS and $N$ is the number of NS in the volume $V$.

The solutions were then sonicated for 2 hours in order to ensure a good dispersion of the NS.

The solutions were deposited on fused silica surface (15 $\times 15 \mathrm{~mm}$ ) purchased from Nayco. These substrates display high $\mathrm{OH}$ content and high flatness. Prior to the deposition of the NS, the substrates were cleaned with acetone and ethanol and treated with UV-ozone for 30 minutes in order to form a hydrophilic surface [17].

The solutions were then spin-coated in a two-step process. The first step is the acceleration of the rotation speed from $0 \mathrm{rpm}$ to $2000 \mathrm{rpm}$. We have investigated the effect of different acceleration durations: $50 \mathrm{~s}, 100 \mathrm{~s}, 150 \mathrm{~s}$, and $200 \mathrm{~s}$. These correspond, respectively, to $4.2 \mathrm{rad} \cdot \mathrm{s}^{-2}, 2.1 \mathrm{rad} \cdot \mathrm{s}^{-2}, 1.4 \mathrm{rad} \cdot \mathrm{s}^{-2}$, and $1.0 \mathrm{rad} \cdot \mathrm{s}^{-2}$. During the second step, the sample rotated at a constant speed $(2000 \mathrm{rpm})$ for $150 \mathrm{~s}$.

All the observations were performed on an SEM Raith Pioneer equipped with a field emission gun (FEG) source. The samples were imaged at $3 \mathrm{kV}$.

\section{Results and Discussion}

The spreading of silica NS in DMF solutions by spin coating was tested. Single layers of closely packed NS were obtained without any optimization. The experiment was carried out for NS of $310 \mathrm{~nm}$ and $540 \mathrm{~nm}$ diameter for an acceleration duration of $150 \mathrm{~s}$ and a volume fraction of, respectively, $6.1 \%$ and $7.0 \%$ (Figures 1(a) and 1(b)). The quality of the organization for both diameters can be seen on the micrometer scale thanks to the SEM images. At a larger scale, Bragg reflection can be seen confirming the organization on the whole sample surface.

The experiment was repeated for different volume fractions and acceleration. For low concentration and high acceleration, single layers are observed displaying some vacancies. When the concentration of NS is increased, complete double layers can be observed for both sizes of NS. In Figure 2, the cross sections of two samples are shown. Both samples are made of $540 \mathrm{~nm}$ NS solutions with a volume fraction of $7 \%$. In order to obtain a double layer, the acceleration was decreased from $2.1 \mathrm{rad} \cdot \mathrm{s}^{-2}$ to $1.0 \mathrm{rad} \cdot \mathrm{s}^{-2}$.

A set of acceleration and concentration has been tested for both diameters in order to identify the optimal parameters allowing the deposition of single or double layers. The surface coverage was measured by analyzing 20 adjacent SEM scans of $75 \mu \mathrm{m} \times 50 \mu \mathrm{m}$. The number of layers is represented in Figure 3(a) for the $310 \mathrm{~nm}$ NS and in Figure 3(b) for the 540 nm NS. " 1 " indicates a single layer obtained for a given volume fraction and given acceleration, while " 2 " stands for a double layer. The fractional numbers refer to the proportion of double layers on the surface. For example, 1.7 means that $30 \%$ of the surface is covered by a single layer while $70 \%$ is covered by a double layer.

The diagrams in Figures 3(a) and 3(b) reflect the ease of getting a single layer of silica NS since, for volume fractions 


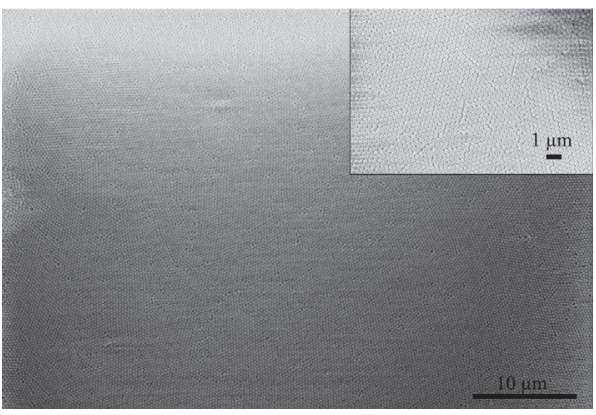

(a)

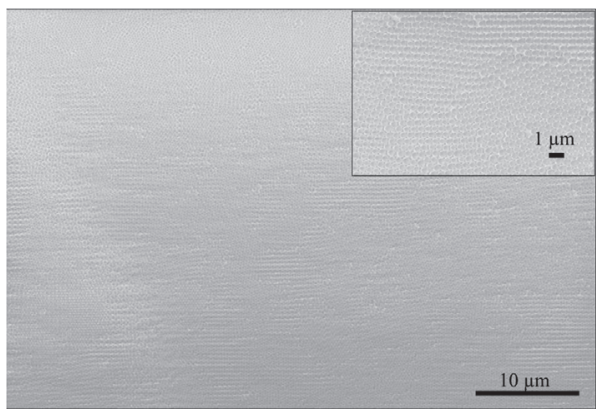

(b)

Figure 1: Single layers of NS of (a) $310 \mathrm{~nm}$ and (b) $540 \mathrm{~nm}$ diameter obtained for acceleration of $1.4 \mathrm{rad} \cdot \mathrm{s}^{-2}$ and volume fractions of (a) $6.1 \%$ and (b) $7.0 \%$.

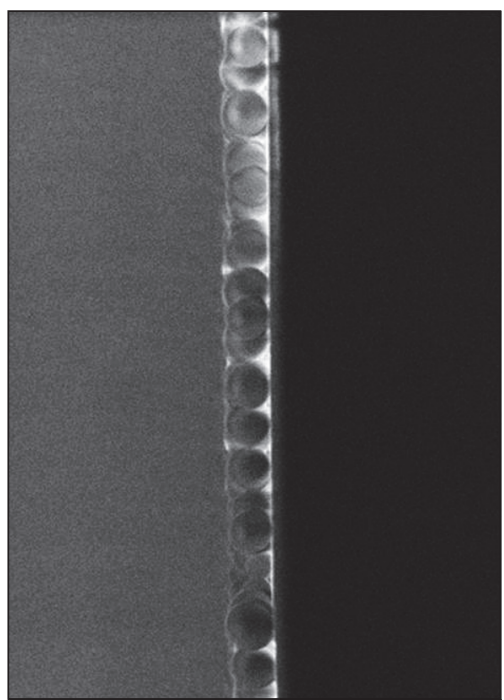

(a)

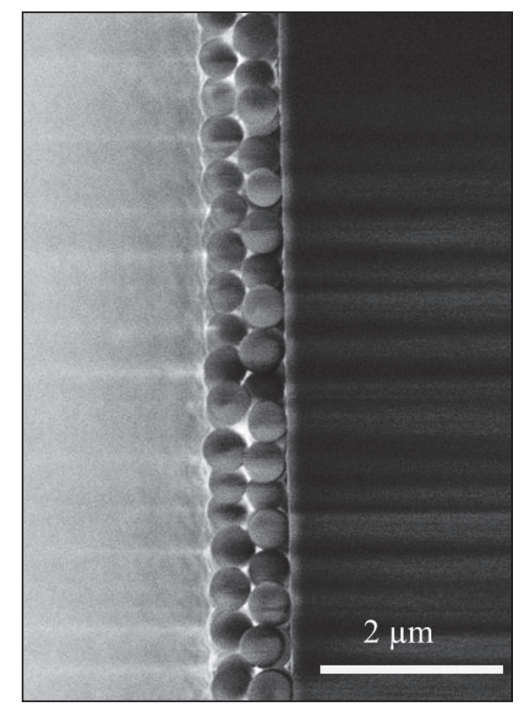

(b)

FIgURE 2: Cross section of a sample obtained by the deposition of NS of $540 \mathrm{~nm}$ diameter for a volume fraction of $7.0 \%$ and acceleration of (a) $2.1 \mathrm{rad} \cdot \mathrm{s}^{-2}$ and (b) $1.0 \mathrm{rad} \cdot \mathrm{s}^{-2}$.

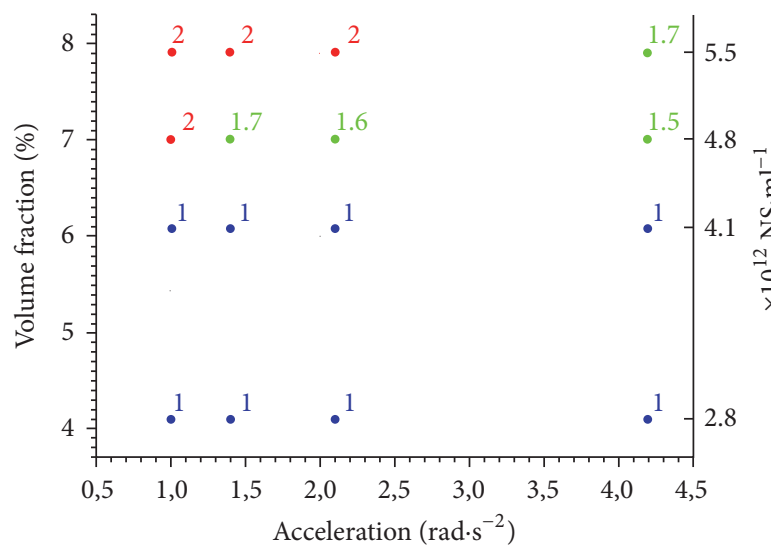

(a)

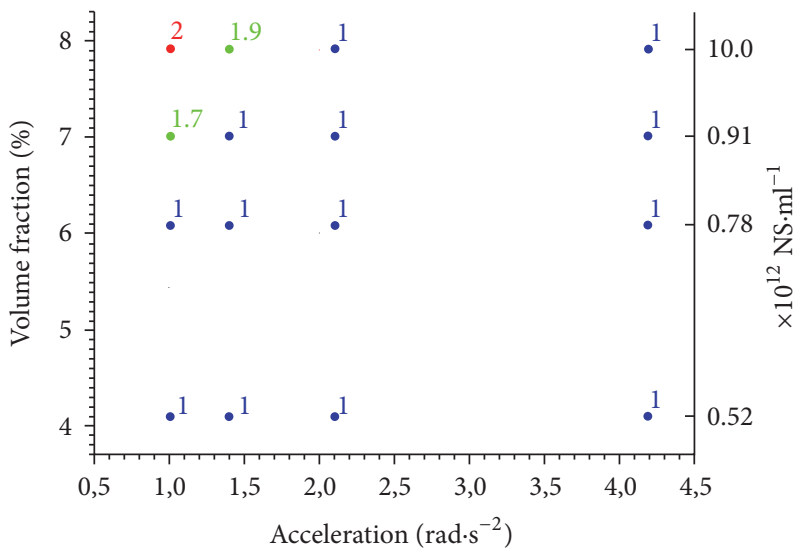

(b)

FIGURE 3: Number of layers of self-organized NS as a function of the volume fraction and the acceleration. "1" indicates that a single layer was observed on the entire surface of the sample; " 2 " indicates that a double layer of organized NS was found on the whole sample surface. Noninteger numbers represent the proportion of double layers on these samples of NS of (a) $310 \mathrm{~nm}$ and (b) 540 nm diameter. 

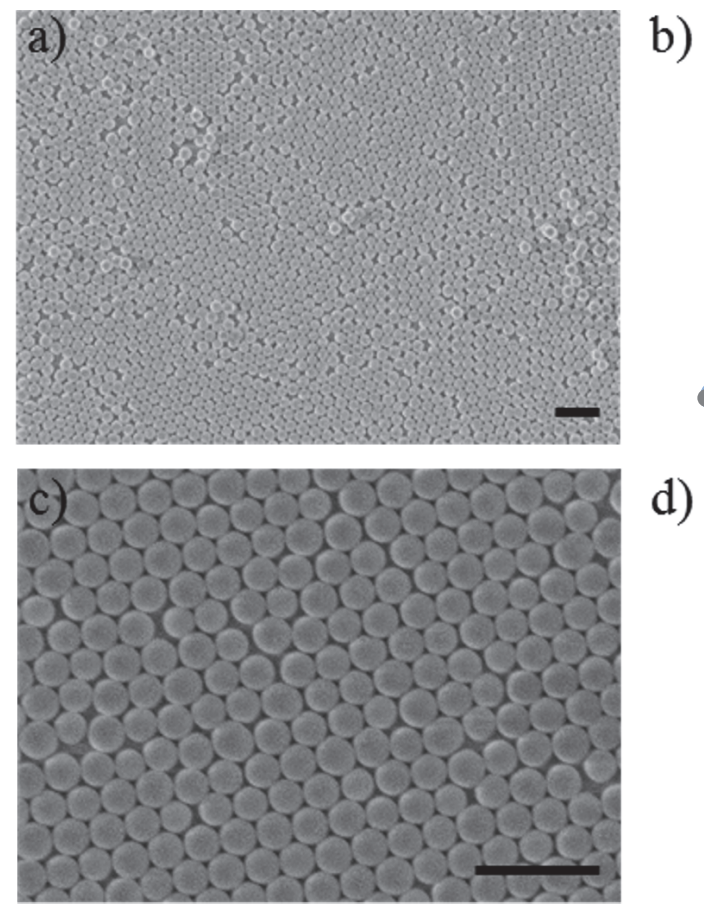

d)
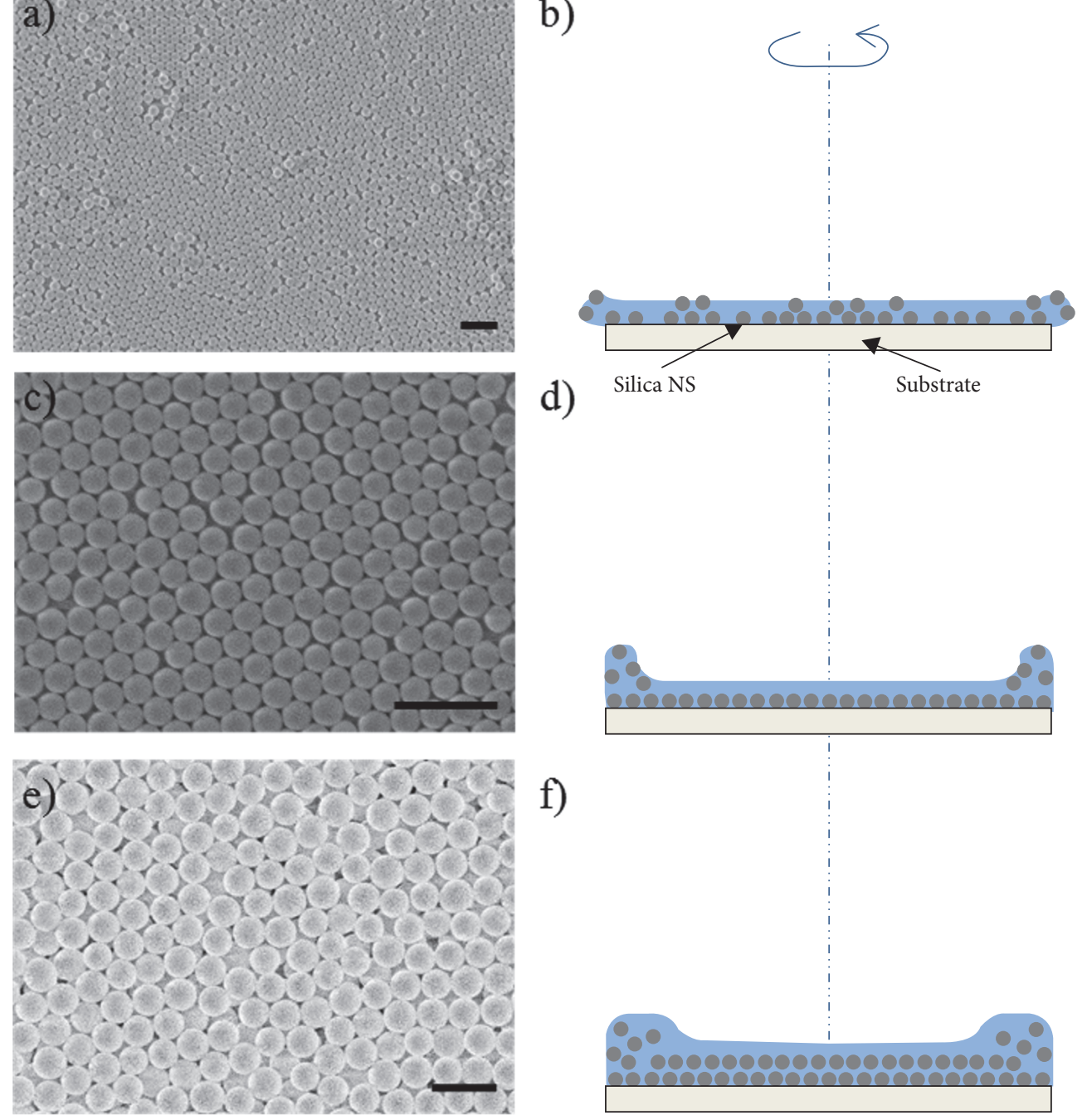

FIGURE 4: Formation of silica NS layers by spin coating. (a, c, e) SEM scan of silica NS spin-coated on fused silica for $4.2 \mathrm{rad} \cdot \mathrm{s}^{-2}, 2.1 \mathrm{rad} \cdot \mathrm{s}^{-2}$, and $1 \mathrm{rad} \cdot \mathrm{s}^{-2}$ and volume fraction of $4.1 \%, 6.1 \%$, and $7.9 \%$. The scale bars stand for $1 \mu \mathrm{m}$. (b, d, f) Corresponding schematics of the process of spreading during spinning.

of $4.1 \%$ and $6.1 \%$, single layers are obtained for both sizes and for all the acceleration values tested. A more complex behavior is observed for the volume fraction of $7.0 \%$ and $7.9 \%$. As the volume fraction increases, multilayers of NS are observed. In the first step, the NS are organized in single layers partly covered by a second one. Then, for other samples, well organized double layers are observed. One can notice that, for a given volume fraction, the proportion of double layers becomes higher when the acceleration is lowered (see, e.g., in Figure 3(b) the volume fraction of $7.9 \%$ giving a double layer for $1 \mathrm{rad} \cdot \mathrm{s}^{-2}, 1.9$ layers for $1.4 \mathrm{rad} \cdot \mathrm{s}^{-2}$, and only one layer for $2.1 \mathrm{rad} \cdot \mathrm{s}^{-2}$ ). Also, samples displaying double layers (partly or entirely) are more often encountered for the $310 \mathrm{~nm}$ diameters rather than for the $510 \mathrm{~nm}$. Controlling the number of layers on the surface by adjusting the acceleration, the volume fraction, and the size of the NS appears to be possible.

The acceleration allows the solution to spread on the substrate surface. This is probably during this period of time when the organization of the NS occurs [12]. As the NS layers are formed, the excess solvent forms bulges on the sample borders. These bulges are expelled when the sample reaches its maximum speed. During this second period, the evaporation of the solvent takes place. If the acceleration is too high, the bulges are immediately expelled and there is no time for the NS to self-organize. This results in a single layer displaying vacancies (Figures 4(a) and 4(b)). Lower acceleration allows the organization of the NS inside the thin layer of the solution between the bulges (Figures 4(c) and 4(d)). The capillary forces play an important role in packing the NS together; if the acceleration is too high, the centrifugal force will exceed the capillary forces and the packing will be poor. If the acceleration is too low, the packing of NS will be better and can even result in a multilayer (Figures 4(e) and $4(f)$ ). Since DMF has a low surface tension coefficient (about $\left.35 \mathrm{mN} \cdot \mathrm{m}^{-1}[18]\right)$, it spreads perfectly on hydrophilic surfaces. The bulges are reduced and a uniform concentration of NS is present on the whole sample surface. Yet, these facts fail 
to explain the difference of behavior encountered for $310 \mathrm{~nm}$ and $540 \mathrm{~nm}$ NS.

Regarding the differences in the two diagrams of Figure 3, one needs to take into account two properties of the NS solutions: the NS sedimentation and the viscosity. This latter parameter is linked to the surface tension of the solution that keeps the NS together despite the spinning and the sedimentation. We propose a mechanism for the formation of organized layers by spin coating of silica NS dispersed in DMF based on the competition between sedimentation of the NS and the increasing viscosity of the solution. The sedimentation speed of NS is well described by the following equation:

$$
v=\frac{2}{9} \frac{R^{2} \Delta \rho g}{\eta},
$$

where $R$ is the sphere radius, $\Delta \rho$ is the density difference between silica and solvent, $g$ is the acceleration of gravity, and $\eta$ is the viscosity of the solvent.

This gives a sedimentation speed of $97 \mathrm{~nm} \cdot \mathrm{s}^{-1}$ and $290 \mathrm{~nm} \cdot \mathrm{s}^{-1}$, respectively, for the $310 \mathrm{~nm}$ and $540 \mathrm{~nm} \mathrm{NS}$ before spinning starts. As the volume fraction increases, the interaction between the NS becomes more important. This leads to a more viscous behavior when the volume fraction becomes larger [19]. As a consequence, the sedimentation speed of the NS inside the solution is lower. In such solution, the sedimentation is not the main phenomenon anymore. Smaller NS display a greater number in the solution for the same volume fraction (right $y$-axis in the diagrams in Figure 3). This explains why the viscous regime is reached for a lower volume fraction.

In order to control the number of layers when depositing NS on a surface, a trade-off has to be found between a sedimentation dominated regime and a viscosity dominated regime. Thus, to obtain a multilayer sample, the volume fraction of the $310 \mathrm{~nm}$ NS has to be increased. Another possibility would be to reduce the size of NS, but then the sedimentation would be much slower and the good compromise even more difficult to find.

\section{Conclusion}

In conclusion, we have proposed a protocol to deposit and control the number of layers of self-organized silica NS on surfaces. The surface of silica that we have used is completely covered by these organized layers, and we believe that it could easily be transferred to even larger surfaces. A mechanism for the formation of these structures is also proposed. The benefit of DMF is found in its high wettability which allows the almost perfect coverage of the substrate and its evaporation rate which is low enough to improve the self-organization of the NS. We found that the competition between the sedimentation and the viscosity of the solution is at the root of the formation of single or double layers. Smaller NS will increase the viscosity of the solution more efficiently and thus decrease the sedimentation. Large-scale double layers of silica NS will then be easier to obtain with smaller diameters. However, it would probably be challenging to make multilayer samples. For NS of $310 \mathrm{~nm}$, the nanopowder of silica NS will reach the limit of dispersion before the appropriate volume fraction could be reached. Jiang and McFarland [13] circumvented this problem by introducing a polymer matrix in the solvent. This increased the viscosity of the solution and formed multilayer samples.

\section{Conflicts of Interest}

The authors declare that they have no conflicts of interest.

\section{Acknowledgments}

The authors would like to acknowledge the "Institut Interdisciplinaire en Sciences Expérimentales" of Université Paris 13 for the financial support.

\section{References}

[1] S. Y. Chou, P. R. Krauss, and P. J. Renstrom, "Nanoimprint lithography," Journal of Vacuum Science \& Technology B, Nanotechnology and Microelectronics: Materials, Processing, Measurement, and Phenomena, vol. 14, no. 6, pp. 4129-4133, 1996.

[2] M. Cottat, N. Lidgi-Guigui, I. Tijunelyte et al., "Soft UV nanoimprint lithography-designed highly sensitive substrates for SERS detection," Nanoscale Research Letters, vol. 9, no. 1, 2014.

[3] O. Seitz, M. M. Chehimi, E. Cabet-Deliry et al., "Preparation and characterisation of gold nanoparticle assemblies on silanised glass plates," Colloids and Surfaces A: Physicochemical and Engineering Aspects, vol. 218, no. 1-3, pp. 225-239, 2003.

[4] O. Péron, E. Rinnert, M. Lehaitre, P. Crassous, and C. Compère, "Detection of polycyclic aromatic hydrocarbon (PAH) compounds in artificial sea-water using surface-enhanced Raman scattering (SERS)," Talanta, vol. 79, no. 2, pp. 199-204, 2009.

[5] F. Járai-Szabó, S. Aştilean, and Z. Néda, "Understanding selfassembled nanosphere patterns," Chemical Physics Letters, vol. 408, no. 4-6, pp. 241-246, 2005.

[6] B. Van Duffel, R. H. A. Ras, F. C. De Schryver, and R. A. Schoonheydt, "Langmuir-Blodgett deposition and optical diffraction of two-dimensional opal," Journal of Materials Chemistry, vol. 11, no. 12, pp. 3333-3336, 2001.

[7] M. Bardosova, P. Hodge, L. Pach et al., "Synthetic opals made by the Langmuir-Blodgett method," Thin Solid Films, vol. 437, no. 1-2, pp. 276-279, 2003.

[8] N. Lidgi-Guigui, C. Dablemont, D. Veautier et al., "Grafted 2D assembly of colloidal metal nanoparticles for application as a variable capacitor," Advanced Materials, vol. 19, no. 13, pp. 17291733, 2007.

[9] M. Szekeres, O. Kamalin, P. G. Grobet et al., "Two-dimensional ordering of Stöber silica particles at the air/water interface," Colloids and Surfaces A: Physicochemical and Engineering Aspects, vol. 227, no. 1-3, pp. 77-83, 2003.

[10] G. T. Ayenew, A. P. A. Fischer, C.-H. Chan et al., "Self-organized nanoparticle photolithography for two-dimensional patterning of organic light emitting diodes," Optics Express, vol. 22, no. 21, pp. A1619-A1633, 2014.

[11] J.-Y. Choi, T. L. Alford, and C. B. Honsberg, "Solvent-controlled spin-coating method for large-scale area deposition of twodimensional silica nanosphere assembled layers," Langmuir, vol. 30, no. 20, pp. 5732-5738, 2014. 
[12] T. Ogi, L. B. Modesto-Lopez, F. Iskandar, and K. Okuyama, "Fabrication of a large area monolayer of silica particles on a sapphire substrate by a spin coating method," Colloids and Surfaces A: Physicochemical and Engineering Aspects, vol. 297, no. 1-3, pp. 71-78, 2007.

[13] P. Jiang and M. J. McFarland, "Large-scale fabrication of wafersize colloidal crystals, macroporous polymers and nanocomposites by spin-coating," Journal of the American Chemical Society, vol. 126, no. 42, pp. 13778-13786, 2004.

[14] C. L. Haynes and R. P. Van Duyne, "Nanosphere lithography: a versatile nanofabrication tool for studies of size-dependent nanoparticle optics," The Journal of Physical Chemistry B, vol. 105, no. 24, pp. 5599-5611, 2001.

[15] L. A. Dick, A. D. McFarland, C. L. Haynes, and R. P. Van Duyne, "Metal film over nanosphere (MFON) electrodes for surfaceenhanced Raman spectroscopy (SERS): improvements in surface nanostructure stability and suppression of irreversible loss," The Journal of Physical Chemistry B, vol. 106, no. 4, pp. 853-860, 2002.

[16] J. C. Hulteen, D. A. Treichel, M. T. Smith, M. L. Duval, T. R. Jensen, and R. P. Van Duyne, "Nanosphere lithography: size-tunable silver nanoparticle and surface cluster arrays," The Journal of Physical Chemistry B, vol. 103, no. 19, pp. 3854-3863, 1999.

[17] J. R. Vig and J. W. L. Bus, "UV/Ozone Cleaning of Surfaces," IEEE Transactions on Parts, Hybrids, and Packaging, vol. 12, no. 4, pp. 365-370, 1976.

[18] H. Kahl, T. Wadewitz, and J. Winkelmann, "Surface tension of pure liquids and binary liquid mixtures," Journal of Chemical \& Engineering Data, vol. 48, no. 3, pp. 580-586, 2003.

[19] J. C. van Der Werff and C. G. De Kruif, "Hard-sphere Colloidal Dispersions: The Scaling of Rheological Properties with Particle Size, Volume Fraction, and Shear Rate," Journal of Rheology, vol. 33, no. 3, pp. 421-454, 1989. 


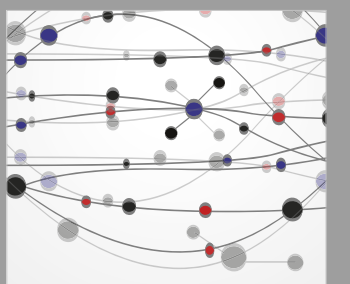

The Scientific World Journal
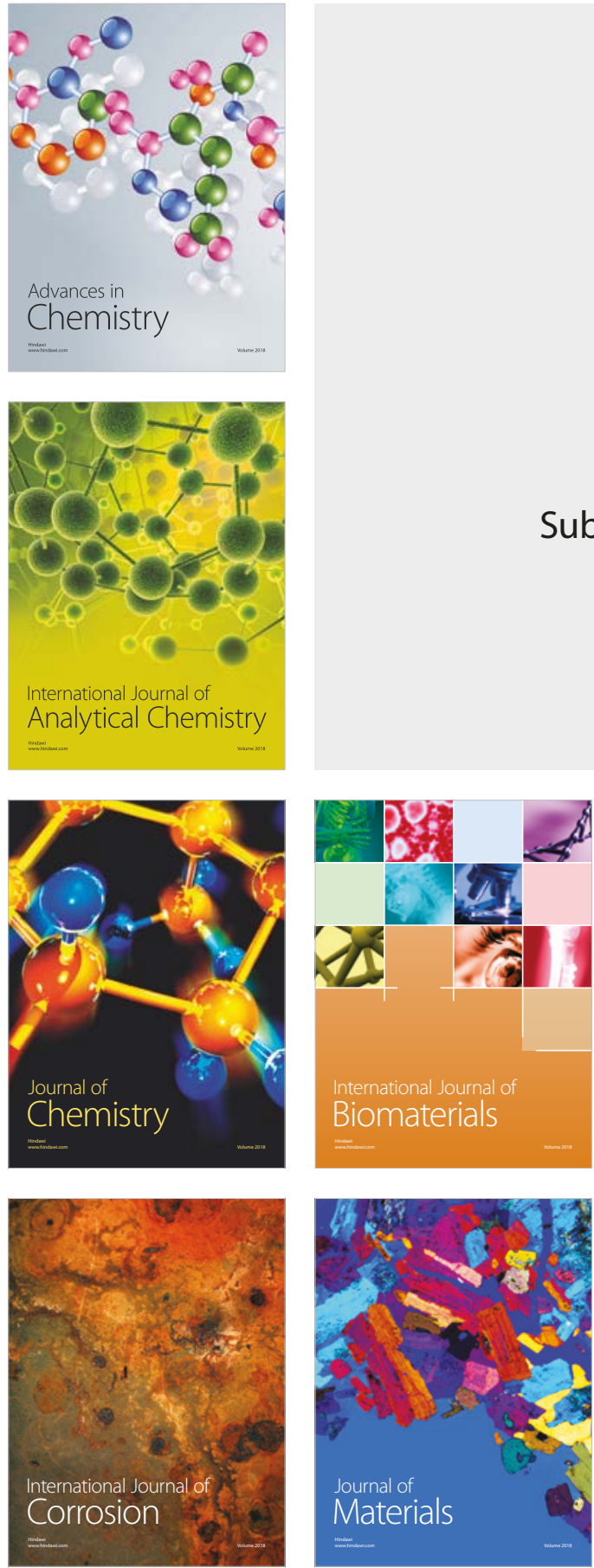

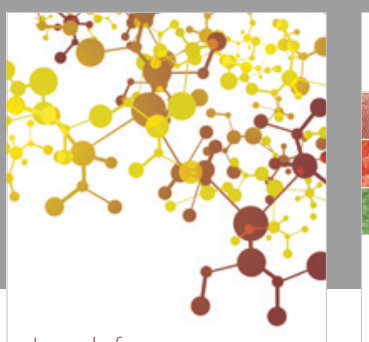

Journal of

Applied Chemistry
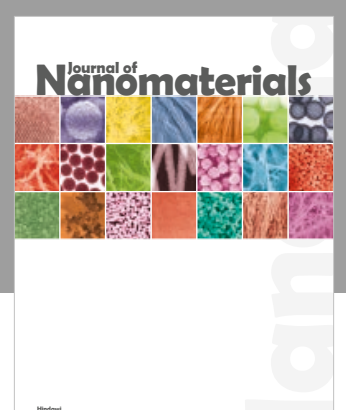

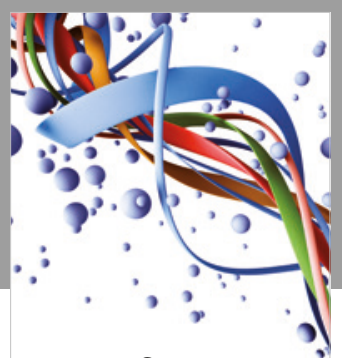

Scientifica

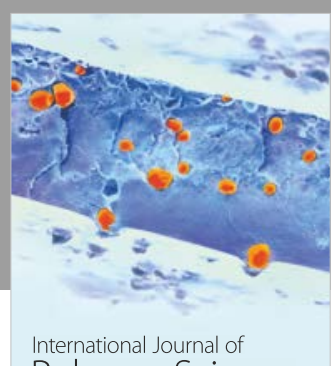

Polymer Science

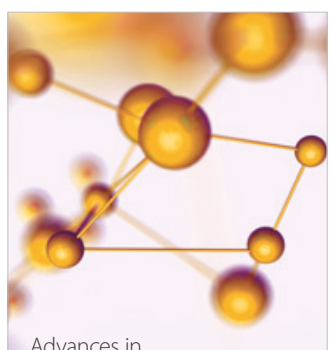

Physical Chemistry
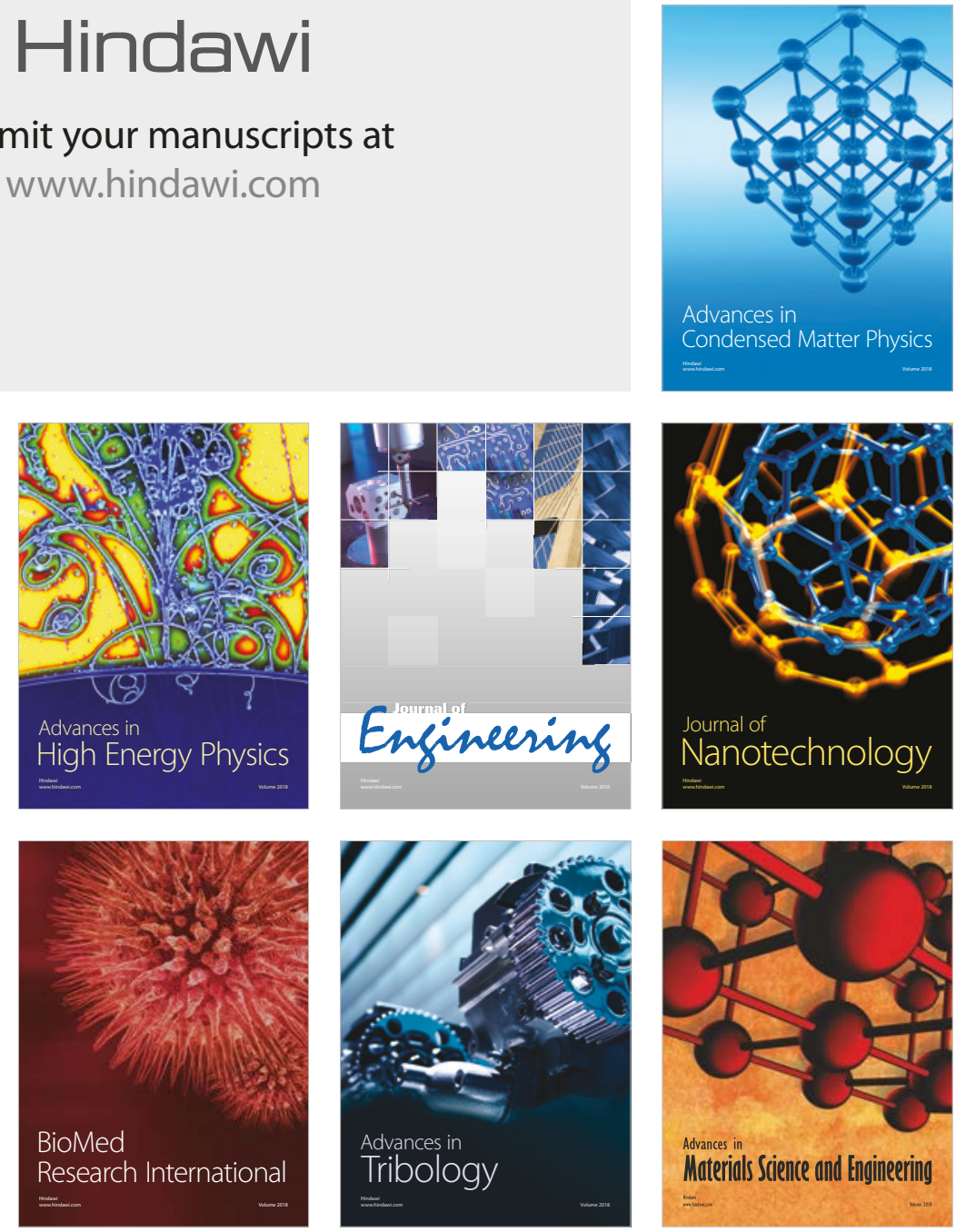